\title{
Path Planning-aiding System of Unmanned Aerial Vehicle in Freight Transportation
}

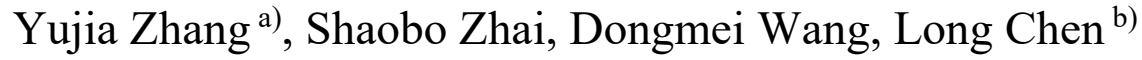 \\ School of Automation Northwestern Polytechnical UniversityXi'an, China, 710072, China. \\ a) Corresponding author: zyjagllfb@163.com \\ b) caidelong@mail.nwpu.edu.cn
}

\begin{abstract}
This paper deals with the Unmanned Aerial Vehicle (UAV) Routing and Scheduling problem applied in logistics, with the implementation of Dynamic Mixed Integer Linear Programming (MILP) to tackle the problem. The MILP accounts for multiply constraints and objectives based on the demand of practicality and flexibility in real logistic operation: the minimal traveling cost and shortest delivery time, division of cold chain and ordinary logistics, aerial vehicle capacity, delivery and pickup requirement, the damage rate of cold chain goods, time window, etc. Formulations are proposed by modifying the vehicle routing problem with time window (VRPTW) with the increased time and space complexity. Then computer experiment is carried out by applying a commercial linear equation solver Gurobi. The output of proposed model involves the optimal routes, which shows a highly-acceptable solution with high-efficiency.
\end{abstract}

Key words: UAV; MILP; VRPTW; time window; commercial linear; highly-acceptable.

\section{INTRODUCTION}

In October 2017, the Federal Aviation Administration of United States announced the UAS Integration Pilot Program, to integrate the Unmanned Aerial Vehicle (UAV) flight system into the American national airspace system and further promote the navigation of unmanned aircraft. The contents involve freight transportation by UAV, construction of UAV navigation network, etc. Before that, UAV logistics project had been blocked in companies of America due to the restrictions of U.S. government, Amazon for illustration, which means this program is of great significance to accelerate the development of UAV freight transportation.

Besides, DHL in Germany, working in drone delivery for years, extended their business to more comprehensive programs involving multi-modal transportation, warehousing and distribution activities by UAV. FPS in UK, in 2015 the company completed the first UK B2B delivery in Shaffield made by a drone. Within the drone delivery industry, the number of such enterprises is of sharp rising in recent years, which means to create a UAV freight transportation network and operate in full commercial pattern is no longer too far away. Here the basic principle to operate the delivery drones is to make routing and scheduling of flight drones in order to satisfy the customer needs, considering obstacles, energy loss and limited capacity, the UAV freight transportation can be optimized with high efficiency thus less labor is needed and no longer subject to the traffic condition. In view of such problem, this paper presents an approach for UAV route scheduling based on reality. The problem is formulated as a mixed integer linear programming model for purpose of minimizing the total cost of aerial vehicle routes for serving all clients in different distribution centers with their demands.

The outline of this paper is organized as follows. Section II presents the existing literature on UAV delivery and the novelty of our study. Section III states the definition and mathematical formulation of the problem. Section IV illustrates the numerical results based on a commercial program solver Gurobi with the test instances derived from Solomon benchmarks. Section V concludes the conducted research and provides suggestions of future studies. 


\section{LITERATURE REVIEW}

Path planning for unmanned aerial vehicle is widely applied to survey mapping, reconnaissance mission, also essential to the drone freight transportation. Such planning and scheduling can be seen as a complex problem because the flight trajectory is affected by multiply factors, proving to be a hybrid process which involves both operational research (OR) and dynamics. In most literatures, ignore of space representation, by the comparison of similarity between drone delivery and trunk delivery, the UAV routing and scheduling problem is categorized as a special case of combinatorial optimization problem, with traveling salesman problem (TSP) [1] and vehicle routing problem (VRP) [2] included. Some literatures also consider it as the variant of the generalized assignment problem (GAP) [3]. Generally, such UAV navigation system is usually formulated in MILP model which is inspired by T.Schouwenaars to solve combinatorial optimization problems [4]. Bellingham proposed the solution of coordination and control of UAV Fleets using MILP to tackle the allocation and trajectory design problems [5]. Efficient heuristics are also put forward to obtain the optimal results. Among these solutions, song et al. [6] proposed the receding horizon task assignment heuristic together with the sequential task assignment heuristic to quickly derive optimal solutions. Yet most MILP models consider the storage electricity of unmanned aerial vehicle under the ideal state without considering some realistic factors like the energy losses during the flight process.

In recent literatures, at an operational level, to improve the maneuverability of making path planning of UAV, some literatures considered various possible constraints and tackled it with heuristics and meta-heuristics. Among the literatures, Tal Shima took the simultaneous tasks of UAV into consideration, with genetic algorithm (GA) applied for efficient solution searching and Monte Carlo simulations carried out to verify the validity of the algorithm [7]. As for the possible energy shortage of UAV, Coelho, Bruno N., et al. designed a novel real-time UAV scheduling algorithm with multiple charging stations using improved matheuristic algorithms [8]. Some UAV path planning is designed for green purpose, like the reduction of $\mathrm{CO}_{2}$, etc [9]. In consideration of complicated flight environment, a generic framework is proposed by Ma, et al. to integrate an autonomous obstacle detection pattern and reinforcement learning (RL) module, with the principle of imitating human visual detection system and avoid the obstacles [10]. However, few literatures work on the UAV path planning applied in flight transportation, which is capable to serve this recently emergent industry for operational purpose.

In this paper, routing and scheduling model of UAV delivery will be implemented, some realistic factors in flight transportation will be discussed, time window of customer delivery requirement, cold chain and ordinary logistics, UAV capacity, the damage rate of cold chain commodity, etc. The problem is formulated in MILP model and solved with a commercial linear problem solver Gurobi.

\section{MATHEMATICAL FORMULATION}

In this study, we denote $G=(V, A)$ to be a set of spatial distribution and the flight traveling arc. The position coordinate is denoted as $\mathrm{V}$, involving the distribution center, depot and refrigerated warehouse, formulated as $V=\{1, \ldots, \boldsymbol{n}\} \bigcup\{\boldsymbol{d}\} \bigcup\{\boldsymbol{r}\}$. The set of arc A equals to $\{(i, j): i, j \in V, i \neq j\} . X_{i j}$ is a binary variable that equals to one if the drone visits $j$ after leaving the node $i$, zero otherwise The $\operatorname{arc}(i, j) \in A$ corresponds to the distance $d_{i j}$ between the node $\mathrm{i}$ and $\mathrm{j}$, also binds with the traveling time $t_{i j}$. Nodes $N=\{1, \ldots, n\}$ correspond to logistics distribution centers. Node $d$ represents the depot and node $r$ represents the refrigerated warehouse in practice.

Each drone $\mathrm{k} \in \mathrm{K}$ has delivery requirements (cold chain products in cold chain logistics/ordinary goods in conventional logistics). For conventional logistics, let's denote $d_{d i}$ is the quantity of delivery commodities from the depot required by a set of the responsible clients of logistics distribution center i. Then aerial vehicles will go to the next distribution center directly after departing from depot. Simultaneously, for the general system mode of cold chain logistics, some material requires low temperature storage which is only available in the refrigerated warehouse. Under this condition the vehicles must visit the warehouse after leaving from the depot the go to the next distribution center. Here we denote that $d_{r i}$ represents the quantity of special items required by clients $i$ from refrigerated warehouse. To be more concise, we mark these two types of delivery demands as delivery 1 and delivery 2. $P_{r} \in \mathrm{N}$ denotes the set of logistics distribution centers requiring the delivery from the refrigerated warehouse. $z_{i}$ is the binary decision parameter which equals to one if the logistics is of cold chain, zero otherwise Based on practical the cold chain products corresponding to a damage rate, assume that the aerial vehicle maintains 
a constant temperature during delivery process and the damage rate is fixed. Denote $\theta \mathrm{r}$ as the parameter for damage rate per kilometer, parameter $\gamma \mathrm{r}$ marches with the per-unit price of the commodity.

Besides the two types of delivery requirement, every distribution center also has pickup requirements (consider that some clients are likely to send parcel post, in studied model these packages will return to depot first). Define that $p_{d i}$ is the parameter governing the quantity of pickup commodities required by clients $\mathrm{i}$, with the hypothesis that all pickup commodities are transported to depot without sending to refrigerated house. Define the quantity of pickup commodities is $q\left(p_{i j k}\right)$. If drone $\mathrm{k}$ flies from $\mathrm{i}$ to $\mathrm{j}$, the quantity of delivery commodities is $q\left(d_{i j k}\right)$.

The requirement of each distribution center is associated with a time window $\left[t_{a i}, t_{b i}\right]$, where $t_{a i}$ represents the earliest time and the $t_{b i}$ is the latest visiting time. The UAV is permitted to arrive at the distribution center $i$ before $t_{a i}$ but not allowed to arrive after $t_{b i}$. The actual arriving time at node $\mathrm{i}$ of aerial vehicle $\mathrm{k}$ is denoted as $t(s)_{k i}$. The processing time at node $\mathrm{i}$ is assumed to be an identical value $\tau$, which includes the descending time, unloading and loading time and ascending time. The journey time between node $\mathrm{i}$ and node $\mathrm{j}$ is denoted as $t_{i j k}$, the processing time $\tau$ is included in $t_{i j k}$, and the corresponding distance between these two node $\mathrm{i}$ and $\mathrm{j}$ is $d_{i j k}$. Assume that the flying speed of the aerial vehicle is a constant parameter $\mathrm{v}$.

Supposing a fleet $\mathrm{K}$ of identical aerial vehicles with capacity $\mathrm{C}$ are available to serve (here $\mathrm{C}=200$ ). The volume of each drone is assumed to be hard constraints which should be strictly obeyed. For every drone $\mathrm{k} \in \mathrm{K}$, it carries out at most one route. For the visiting command from the distribution centers, each center is visited by exactly one aerial vehicle during distribution, but each vehicle can serve different distribution centers during the planning horizon. The problem lies in determining the flight path with the minimal total cost measured by both time and expenditure. Here we assume that the expenditure per unit distance is measured by $\alpha$, which represents the charging cost per kilometer. Note that for UAV, we should consider the maximum energy that the battery could provide when making ahead scheduling, with $w_{i k}$ denoted as the general consumed energy when flying the entire route at the node $i$. We also define a parameter $p_{i j k}$ as the average power as aerial vehicle flying from node $\mathrm{i}$ to load $\mathrm{j}$. Assuming that to satisfy energy requirement, operators will provide full-charged battery for UAV, with $s_{k}$ defined as the initial full- charged battery energy. The parameter $\mathrm{M}$ is considered to be a large positive number:

Here we also make a list of rules considered in our model:

1. We do not consider recharging battery during the process of delivery, from the cost perspective it won't bring any savings to a drone-delivery company considering the capital investment of rechargeable station.

2. The impact of weather condition on UAV energy consumption is ignored.

3. The UAV fly trajectory is assumed to be perfect-risk-free path without obstacle.

Objective function:

$$
\operatorname{Min} \sum_{k \in K} \sum_{i \in V} \sum_{j \in V}\left(\alpha \cdot d_{i j k}+z_{i} \cdot \theta_{r} \cdot \gamma_{r} \cdot t_{i j k}\right) x_{i j k}
$$

Subject to:

$$
\begin{gathered}
\sum_{k \in K} \sum_{j \in V} x_{i j k}=1 \quad \forall i \in N \\
\sum_{j \in V} x_{d j k} \leq 1 \quad \forall k \in K \\
\sum_{i \in V} x_{i d k} \leq 1 \quad \forall k \in K \\
\sum_{j \in V} x_{i j k}=\sum_{j \in V} x_{j i k} \quad \forall i \in N
\end{gathered}
$$




$$
\begin{gathered}
\sum_{k \in K} \sum_{i \in V} q(d)_{i j k}-\sum_{k \in K} \sum_{i \in V} q(d)_{j i k}=d_{d j}+d_{r j} \quad \forall j \in N \\
\sum_{k \in K} \sum_{i \in V} q(p)_{j i k}-\sum_{k \in K} \sum_{i \in V} q(p)_{i j k}=p_{d j} \quad \forall j \in N \\
q(d)_{i j k}+q(p)_{i j k} \leq C \cdot x_{i j k} \quad \forall k \in K, i \in V, j \in V \\
t_{a i} \leq t(s)_{k i} \leq t_{b i} \quad \forall k \in K, i \in V, j \in V \\
t(s)_{k j} \geq t(s)_{k i}+t_{i j}-M\left(1-x_{i j k}\right) \forall i \in V, j \in V, k \in K, i \neq j \\
x_{d r k} \leq 1 \quad \forall k \in K \\
\sum_{i \in P_{r}} z_{i} \cdot x_{d i k} \leq 0 \quad \forall k \in K \\
\sum_{i \in V} q(d)_{r i k}-\sum_{i \in V} q(d)_{d r k}=\sum_{i \in V} \sum_{j \in P_{h}} x_{i j k} \cdot d_{r j} \quad \forall k \in K \\
w_{i k}+p_{i 0 k}\left(d_{i 0 k} / v+\tau\right) \leq s_{k}+M\left(1-x_{i 0 k}\right) \forall k \in K, i \in V, j \in V \\
w_{i k}+p_{i j k}\left(d_{i j k} / v+\tau\right) \leq w_{j k}+M\left(1-x_{i j k}\right) \forall k \in K, i \in V, j \in V
\end{gathered}
$$

The objective function aims to minimize the total cost, including the operating cost per kilometer and the spoilage cost of cold chain goods. Constraints (2) ensure that all distribution centers are visited exactly once. Constraints (3) and (4) denote that each route starts from and ends at the depot. Constraints (5) guarantee the routing flow balance for all the nodes by ensuring all the aerial vehicles leaving a node once visit it. Constraints (6) and (7) represent the quantity equations of delivery and pickup demands. Constraints (8) and (9) impose the aerial vehicle capacity and time window constraints. Constraints (10) ensure the visiting time is under the allotted time window. Constraints (11) and (12) denote that in cold chain logistics each aerial vehicle visits the refrigerated warehouse at most once in its route and the vehicle visits the refrigerated warehouse after leaving the depot. Constraints (13) ensure that the delivery demands of the cold chain storage are loaded at the refrigerated warehouse. Constraints (14) ensure that the total energy consumption along the route is not exceed the battery energy. Constraints (15) denote the energy consumption at the node $\mathrm{j}$ if the aerial visit $\mathrm{i}$ and $\mathrm{j}$ in sequence.

\section{COMPUTATIONAL EXPERIMENTS}

This section presents the results of the optimal routes in computational experiments. Considering the characteristics of logistics distribution, there is no benchmark instance adaptive to our problem. Thus, we derive test instances from benchmarks of Solomon (1987) and use the reconstructed Solomon dataset to do the simulation. These instances are divided into three types according to the distribution of the clients: $C$ type instances (clustered clients), $R$ type instances (randomly located clients), $R C$ type instance (clients partly clustered and partly randomly located).

We select two instances respectively from the above types in order to generate new instances for our test. The model is solved by the application of a commercial linear problem solver Gurobi. The parameters qty(dy1), qty(dy2) denote the quantities of commodities to be delivered to distribution centers from the depot demand(dy1), and the 
refrigerated warehouse demand(dy2), if it is the cold chain logistics. Parameter qty(pu) represents the quantity of commodities to be picked up from the clients in distribution centers.

The computational results are expressed as follows: dist total, $c a p_{\text {remain }}$ and $E_{\text {remain }}$ show the optimal value of total distance, the total remain capacity and the remain energy of UAV which can be used for the transportation from the depot to the refrigerated warehouse. Determining variable time indicates the operational time. Decision variable total cost is the result of objective function which should be minimized. The instances are divided into three groups according to the number of distribution centers.

TABLE 1. Computational results for 10 distribution centers

\begin{tabular}{|c|c|c|c|c|c|c|c|c|c|}
\hline instance & Distribution centers & qty(dy1) & qty(dy2) & qty(pu) & dist $_{\text {total }}$ & cap $_{\text {remain }}$ & $E_{\text {remain }}$ & total cost & time \\
\hline instance $_{1}$ & 10 & 150 & 0 & 0 & 65.7 & 50 & $31.27 \%$ & 44.5551 & $0.66 \mathrm{~s}$ \\
\hline instance $_{2}$ & 10 & 150 & 0 & 150 & 65.7 & 50 & $31.27 \%$ & 47.2922 & $0.68 \mathrm{~s}$ \\
\hline instance $_{3}$ & 10 & 150 & 0 & 250 & 105.4 & 250 & $40.09 \%$ & 78.7963 & $18.07 \mathrm{~s}$ \\
\hline instance $_{4}$ & 10 & 150 & 150 & 0 & 106.4 & 250 & $40.31 \%$ & 76.5888 & $5.39 \mathrm{~s}$ \\
\hline instance $_{3}$ & 10 & 150 & 250 & 0 & 109.9 & 250 & $49.42 \%$ & 82.1605 & $64.69 \mathrm{~s}$ \\
\hline
\end{tabular}

TABLE 2. Computational results for 15 distribution centers

\begin{tabular}{|c|c|c|c|c|c|c|c|c|c|}
\hline instance & $\begin{array}{c}\text { Distribution } \\
\text { centers }\end{array}$ & qty(dy1) & qty(dy2) & qty(pu) & dist $_{\text {total }}$ & cap $_{\text {remain }}$ & Eremain $_{\text {reme }}$ & $\begin{array}{c}\text { total } \\
\text { cost }\end{array}$ & time \\
\hline instance $_{6}$ & 15 & 260 & 0 & 0 & 153.7 & 140 & $59.16 \%$ & 109.7826 & $1.37 \mathrm{~s}$ \\
\hline instance $_{7}$ & 15 & 260 & 0 & 260 & 153.7 & 140 & $59.16 \%$ & 120.0276 & $29.92 \mathrm{~s}$ \\
\hline instance $_{8}$ & 15 & 260 & 0 & 360 & 154.7 & 140 & $59.38 \%$ & 125.1051 & $11.46 \mathrm{~s}$ \\
\hline instances $_{9}$ & 15 & 260 & 260 & 0 & 202.2 & 340 & $69.93 \%$ & 157.9023 & $17.44 \mathrm{~s}$ \\
\hline instance $_{10}$ & 15 & 260 & 360 & 0 & 269.5 & 540 & $84.89 \%$ & 217.9432 & $646.85 \mathrm{~s}$ \\
\hline
\end{tabular}

TABLE 3. Computational results for 20 distribution centers

\begin{tabular}{|c|c|c|c|c|c|c|c|c|c|}
\hline instance & $\begin{array}{c}\text { Distribution } \\
\text { centers }\end{array}$ & qty(dy1) & qty(dy2) & qty(pu) & dist $_{\text {total }}$ & capremain $_{\text {E }}$ & Emain $_{\text {rematal }}$ & $\begin{array}{c}\text { totame } \\
\text { cost }\end{array}$ & time \\
\hline instance $_{11}$ & 20 & 360 & 0 & 0 & 177.7 & 40 & $72.82 \%$ & 130.8732 & $550.24 \mathrm{~s}$ \\
\hline instance $_{12}$ & 20 & 360 & 0 & 360 & 177.7 & 40 & $72.82 \%$ & 148.6404 & $603.24 \mathrm{~s}$ \\
\hline instance $_{13}$ & 20 & 360 & 0 & 410 & 200.3 & 240 & $77.84 \%$ & 170.3260 & $140.54 \mathrm{~s}$ \\
\hline instance $_{14}$ & 20 & 360 & 360 & 0 & 297.5 & 440 & $99.43 \%$ & 248.8492 & $8513.16 \mathrm{~s}$ \\
\hline instance $_{15}$ & 20 & 360 & 410 & 0 & 297.5 & 440 & $99.43 \%$ & 252.9805 & $102600.57 \mathrm{~s}$ \\
\hline
\end{tabular}

The computational results of instances with 10, 15 and 20 distribution centers are shown in the following three tables. The instances of the tables are derived from basic Solomon instance $\mathrm{C} 101$ by remaining front 10 customers, 15 customers and 20 customers. They are denoted as C101 - 10, C101 - 15 and C101 - 20. The coordinate of the refrigerated warehouse is $(42,45)$ for the 15 instances shown in this section.

Here, instances 1,6,11 reserve the intrinsic demands of basic Solomon instances, which means demand(dy1) of clients in each distribution center equals to the demand given by Solomon instance. And then demand(dy1) are duplicated to demand(pu) in instances $2,7,12$ and to demand(dy2) in instances4,9,14. To generate instances $3,5,8,10,13,15$, we first randomly choose the distribution centers by rate of $\mathrm{c}(\mathrm{c}=0.6$ in these six instances). The demand(pu) of each chosen distribution center equals to 1:5 of the demand given by Solomon instance in instance 3,8,13. The demand(dy2) in instances5,10,15 are generated similarly. These operations make $\mathrm{qty}(\mathrm{dy} 1=\mathrm{dy} 2=\mathrm{pu})$ also represent the distribution of corresponding demand among the distribution center. For example, if qty(dy1) equals to qty(dy2), it means the distributions of demand(dy1) and demand(dy2) are consistent. 


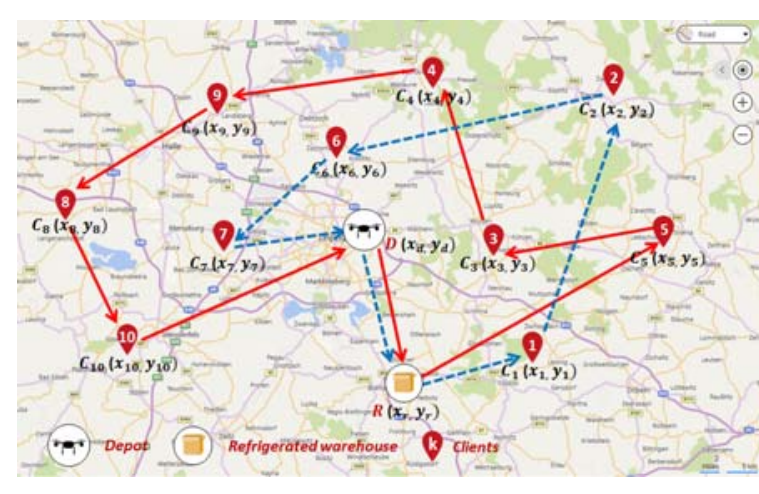

(a)

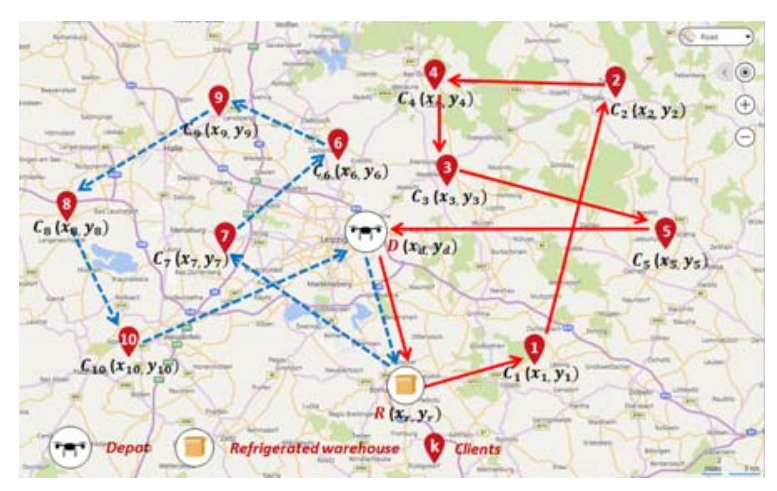

(b)

FIGURE 1. UAV routes for instance 3 and instance 4

Figure 1 (a)(b) are samples for cold chain logistics, which show the UAV routes for the instance 3 and instance 4. The coordinates of nodes are noted in parentheses, for example (x1,y1) and (xr,yr) represent the coordinates of distribution center 1 and refrigerated warehouse. The UAV will start from the depot with all commodities required from the depot, and then visit the refrigerated warehouse before visiting any distribution center. The qty(dy1) in instance 3 and instance 4 are same, but because of the difference of qty(dy2) and qty(pu), the UAV routes for instance 3 an instance 4 are different, although instance 3 and instance 4 have approximate total distance.

The output of the model contains the minimal total cost of all UAV routes, it can help logistics company to make optimal scheduling and better utilization of the existing resources. In order to satisfy the energy constraints, the consumed energy should be no more than the initial energy which is denoted as $100 \%$. Considering the remaining capacity of UAV during the flight, in instance10, the total remaining capacity from the depot to the refrigerated warehouse equals to 540 yet the capacity of each UAV is only 200. It will save the number of UAVs immensely if this remaining capacity can be used for the transportation from the depot to the refrigerated warehouse.

By comparing the result of instance 2 and instance4, instance 7 and instance 9 , instance 8 and instance 10 , instance12 and instance14, with same qty(dy1), the qty(dy2) and qty(pu) are equals in the four pairs of instances. We can see that the quantity of demand(dy2) has more influences to total distance and total remaining capacity of UAVs from depot to refrigerated center, than the quantity of demand(pu), with the remaining energy of storage battery possessing positive correlation with the traveling distance. It is also easily to see from the tables that the increase of quantity of demand(dy2) results in the obvious increase of total distance, operational time and general costs.

\section{CONCLUSION AND FUTURE RESEARCH}

This paper proposes an approach for the unmanned aerial vehicle assignments in freight transportation which can be considered as a special case of vehicle routing problem with time windows (VRPTW). Yet the proposed model is more complex than classical VRPTW for the various constraints of transportation: division of different commodities, energy losses of UAV, delivery and pickup requirement, goods damage rate, etc. Here if regarding the constructed model as a mixed integer linear programming model, solution can be obtained by a commercial linear problem solver Gurobi which proves to have an acceptable operational time in small-scale instances. However, the operational time increases with the increase numbers of distribution centers (instance14 and instance15), thus we can see that the resolution with exact method is not suitable for our problem with large scale instances.

Therefore, inspired by the observed results, future studies will work on treating the large size problem with heuristics and meta-heuristics to reduce the operational time in a reasonable scale. Simultaneously, on account that the delivery drones will encounter obstacles during each flight, thus obstacle avoidance algorithm is necessary when constructing our models. 


\section{REFERENCES}

1. Shima, Tal, S. Rasmussen, and D. Gross. “Assigning Micro UAVs to Task Tours in an Urban Terrain.” IEEE Transactions on Control Systems Technology 15.4(2007):601-612.

2. G. B. Dantzig and R. H. Ramser, The truck dispatching problem, Management Sci., vol. 6, no. 1, pp. 8091, 1959.

3. G. T. Ross and R. M. Soland, A branch and bound algorithm for the generalized assignment problem, Math. Program., vol. 8, no. 1, pp. 91103, 1975.

4. T. Schouwenaars, B. De Moor, E. Feron, and J. How, Mixed integer programming for multi-vehicle path planning, in Control Conference (ECC), 2001 European, pp. 26032608, IEEE, 2001.

5. Bellingham, John Saunders. "Coordination and Control of UAV Fleets using Mixed-Integer Linear Programming." (2002).

6. Song, Byung Duk, J. Kim, and J. R. Morrison.” Rolling Horizon Path Planning of an Autonomous System of UAVs for Persistent Cooperative Service: MILP Formulation and Efficient Heuristics.” Journal of Intelligent \& Robotic Systems 84.1-4(2016):1-18.

7. AIAA. "Assignment of Cooperating UAVs to Simultaneous Tasks using Genetic Algorithms.” (2005).

8. Coelho, Bruno N., et al. "A multi-objective green UAV routing problem." Computers \& Operations Research (2017).

9. Goodchild, Anne, and J. Toy. "Delivery by drone: An evaluation of unmanned aerial vehicle technology in reducing CO 2, emissions in the delivery service industry." Transportation Research Part D Transport \& Environment (2017).

10. Ma, Zhaowei, et al. "A saliency-based reinforcement learning approach for a UAV to avoid flying obstacles." Robotics \& Autonomous Systems (2017). 